\title{
A comprehensive evolutionary theory deduced from thermodynamics
}

\section{Ji-Ming Chen*}

School of Life Sciences and Engineering, Foshan University, Foshan, Guangdong Province, 528225, China

\section{${ }^{*}$ Corresponding author.}

Email: jmchen678@qq.com; Telephone:+86-13854212160.

\begin{abstract}
The current evolutionary theories have remained incomplete, controversial, and stagnant for multiple decades. To solve this issue, we create the concept of carbon-based entities (CBEs) which include methane, amino acids, proteins, organisms, and other entities containing carbon atoms. We deduce from thermodynamics the driving force, the progressive mechanisms, and the major steps of evolution of CBEs, and hence establish a comprehensive evolutionary theory termed the CBE evolutionary theory (CBEET). The CBEET demonstrates that evolution is driven hierarchy-wise by thermodynamics and favors fitness and diversity. It provides novel explanations for origin of life (abiogenesis), macroevolution, natural selection, sympatric speciation, evolution tempos, animal group evolution, and human society development in a comprehensive and comprehensible way. It elucidates that collaboration, altruism, obeying rules with properly increased freedom are important throughout evolution of CBEs. It refutes thoroughly the wrong notion that negative entropy (negentropy) leads to biological order which is distinct from thermodynamic order. It integrates with research advances in multiple disciplines and bridges laws of physics, evolution in biology, and harmonious development of human society.
\end{abstract}

\section{Keywords}

carbon-based entity; driving force; energy; entropy; evolution; fitness; mechanism; natural selection; negentropy; thermodynamics; theory 


\section{Introduction}

Charles Darwin's evolutionary theory, a breakthrough in science, was built on natural selection. However, its definition of natural selection, survival of the fittest, is literally confusing because some individuals which are less fit can also survive [1-3]. Charles Darwin's evolutionary theory was updated in the middle of last century with the Modern Synthesis, which reinterpreted natural selection as gradual changes in gene frequencies of populations because those individuals carrying adaptive mutations are more reproductively successful, as per mathematical modelling and advances in genetics [1-3].

Various research advances challenged the Modern Synthesis in recent decades. Paleontologists revealed that evolution of many species showed the punctuated equilibrium tempo with little change in long geological periods and significant changes in short geological periods; molecular biologists revealed that many molecular mutations are neutral in natural selection, and some mutations occur not randomly; developmental biologists revealed that some acquired epigenetic changes are heritable and important for adaption of organisms [1-14]. Moreover, the Modern Synthesis remains unable to interpret origin of life and macroevolution (i.e. evolution above the species level) [1-3].

Charles Darwin's theory, the Modern Synthesis, and some other evolutionary theories were largely extrapolated from microevolution (evolution within species) [1-16]. This logic is prone to generate controversial and incomplete views, like the fact that different fishes have different views of the river where they live, and no fishes could see the panorama of the river. Although incomplete and controversial, evolutionary theories have remained stagnant for multiple decades [1-3]. Moreover, evolution is in nature a process of thermodynamics, but evolution has not been well explained by thermodynamics $[1-3,17,18]$ (Supplementary Notes).

Here we deduce a comprehensive and comprehensible evolutionary theory whose framework relies on thermodynamics. This theory is termed the CBE evolutionary theory (CBEET), where CBE denotes carbon-based entity. CBEs include some small molecules (e.g. methane and ethanol), middle organic molecules (e.g. amino acids and nucleotides), large organic molecules (e.g. proteins and nucleic acids), and organisms (e.g. bacteria, animals, and plants). CBEs have hierarchies, and large organic molecules are higher-hierarchy CBEs (HHCBEs) compared with middle organic molecules, but they are lower-hierarchy CBEs (LHCBEs) compared with organisms.

The differences between the CBEET and previous theories are outlined in Figure 1 and listed in Table 1. 


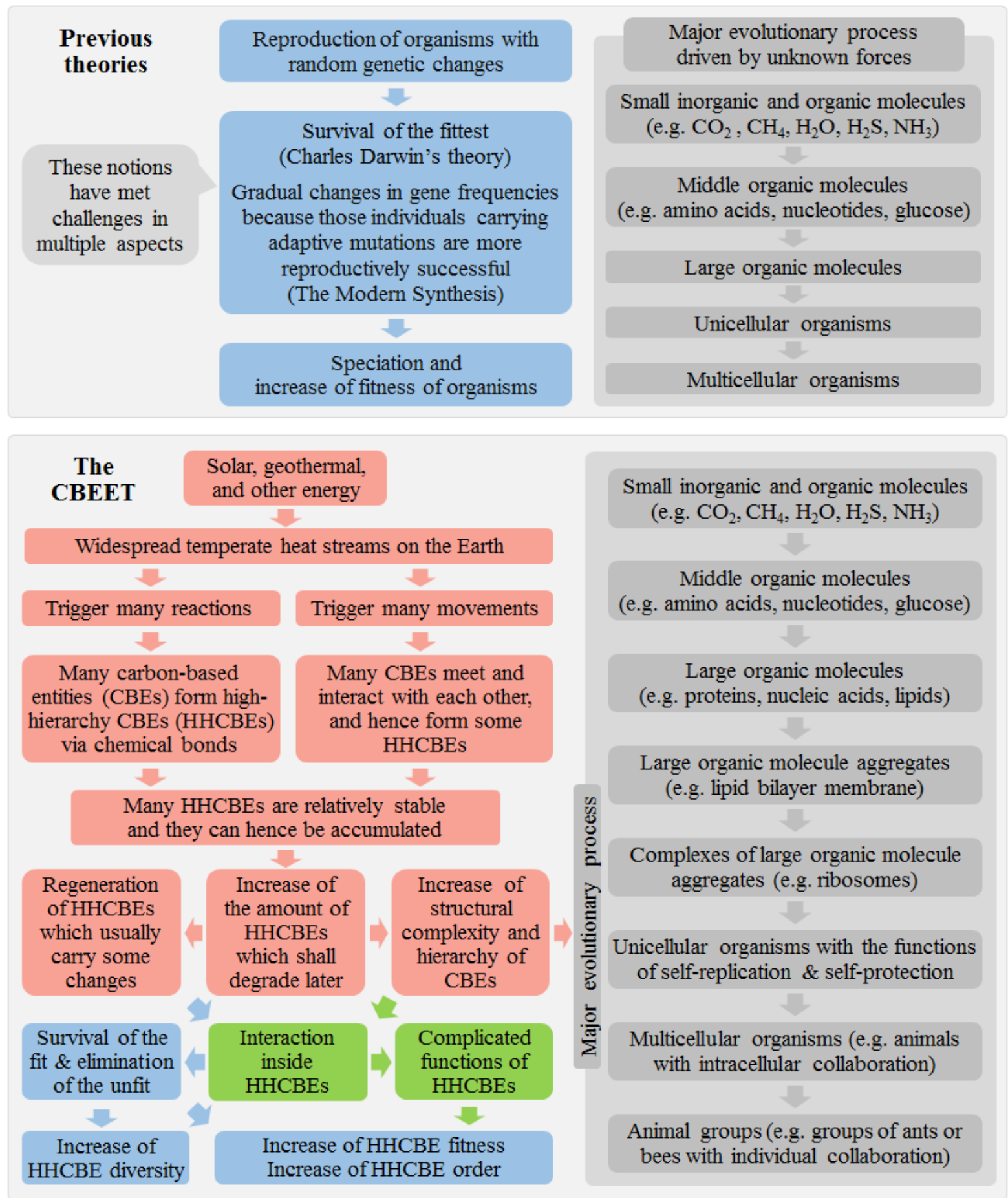

Figure 1. Major views of previous evolutionary theories versus the CBEET. The red, green, and blue arrows represent the driving force mechanism, the structural mechanism, and the natural selection mechanism, respectively. 
Table 1. Differences between previous evolutionary theories and the CBEET.

\begin{tabular}{|c|c|c|}
\hline Aspects & Previous evolutionary theories & The CBEET \\
\hline Logic & $\begin{array}{l}\text { Determining the details and } \\
\text { framework of evolutionary } \\
\text { theories mainly through } \\
\text { extrapolation from microevolution }\end{array}$ & $\begin{array}{l}\text { Determining the details of the theory through } \\
\text { observation of microevolution, and } \\
\text { determining the framework of the theory } \\
\text { through deduction with thermodynamic laws }\end{array}$ \\
\hline $\begin{array}{l}\text { The driving } \\
\text { force of } \\
\text { evolution }\end{array}$ & $\begin{array}{l}\text { atural selection, genetic drift, } \\
\text { utation, or competition (none of } \\
\text { em directly involve energy) }\end{array}$ & $\begin{array}{l}\text { The spontaneous thermodynamic tendency of } \\
\text { carbon-based entities (CBEs) to absorb energy } \\
\text { to form higher-hierarchy CBEs (HHCBEs) }\end{array}$ \\
\hline $\begin{array}{l}\text { Progressive } \\
\text { mechanisms } \\
\text { of evolution }\end{array}$ & $\begin{array}{l}\text { atural selection, sexual selection, } \\
\text { d epigenetic changes lead to } \\
\text { crease of fitness of organisms; } \\
\text { echanisms of macroevolution } \\
\text { main unknown }\end{array}$ & $\begin{array}{l}\text { The driving force increases the structural } \\
\text { complexity and hierarchy of CBEs; CBEs with } \\
\text { increased structural complexity and hierarchy } \\
\text { can obtain spontaneously some complicated } \\
\text { functions; natural selection leads to increase of } \\
\text { diversity and fitness of HHCBEs }\end{array}$ \\
\hline & $\begin{array}{l}\text { Defined as "survival of the fittest" } \\
\text { or "gradual replacement of } \\
\text { populations with those carrying } \\
\text { advantageous mutations"; } \\
\text { highlighting selection in a single } \\
\text { aspect; claiming that mutations } \\
\text { occur randomly and only } \\
\text { inheritable changes are under } \\
\text { natural selection }\end{array}$ & $\begin{array}{l}\text { Defined as "survival of fit HHCBEs and } \\
\text { elimination of unfit HHCBEs"; highlighting } \\
\text { neutral mutations; highlighting the overall } \\
\text { fitness constituted by all traits of HHCBEs; } \\
\text { highlighting selection in various aspects; } \\
\text { accepting that some mutations occur not } \\
\text { randomly, and inheritable changes and } \\
\text { uninheritable changes (e.g. vaccination) are all } \\
\text { under natural selection }\end{array}$ \\
\hline & $\begin{array}{l}\text { Neither the driving force nor the } \\
\text { mechanisms have been proposed } \\
\text { to interpret how large organic } \\
\text { molecules evolved into lives; } \\
\text { stressing autocatalysis and RNA }\end{array}$ & $\begin{array}{l}\text { The driving force and mechanisms of chemical } \\
\text { evolution are revealed; lives originated } \\
\text { hierarchy-wise from small molecules with } \\
\text { several intermediate hierarchies; emphasizing } \\
\text { collaboration of various molecules }\end{array}$ \\
\hline $\begin{array}{l}\text { Biological } \\
\text { evolution }\end{array}$ & $\begin{array}{l}\text { Interpreting sympatric speciation } \\
\text { with diverging selection targeting } \\
\text { different niches in the same area }\end{array}$ & $\begin{array}{l}\text { Proposing a novel sympatric speciation } \\
\text { mechanism: different combinations of various } \\
\text { traits can all constitute adequate fitness } \\
\text { targeting the same niche in the same area }\end{array}$ \\
\hline $\begin{array}{l}\text { Animal } \\
\text { group } \\
\text { evolution }\end{array}$ & $\begin{array}{l}\text { ot having established the } \\
\text { ncept of animal group } \\
\text { olution; difficult to explain } \\
\text { ruism and social norms; having } \\
\text { gative influence on human } \\
\text { ciety }\end{array}$ & $\begin{array}{l}\text { Establishing animal group evolution as the } \\
\text { highest phase of evolution of CBEs; clarifying } \\
\text { that collaboration, altruism, and obeying rules } \\
\text { with properly increased freedom are important } \\
\text { throughout evolution of CBEs; beneficial to } \\
\text { human society development }\end{array}$ \\
\hline $\begin{array}{l}\text { Biological } \\
\text { order and } \\
\text { entropy }\end{array}$ & $\begin{array}{l}\text { Organisms have order and low } \\
\text { entropy due to negative entropy } \\
\text { (input of low-entropy matter and } \\
\text { output of high-entropy matter) }\end{array}$ & $\begin{array}{l}\text { Organisms are of high entropy; the notion of } \\
\text { "negative entropy" is wrong; biological order } \\
\text { is contrary to thermodynamic order; order } \\
\text { should not be always hooked with low entropy }\end{array}$ \\
\hline $\begin{array}{l}\text { General } \\
\text { features }\end{array}$ & $\begin{array}{l}\text { Incomplete, elusive, unable to } \\
\text { integrate with recent discoveries, } \\
\text { separated from physics and social } \\
\text { sciences }\end{array}$ & $\begin{array}{l}\text { Comprehensive, comprehensible, able to } \\
\text { integrate with recent advances in multiple } \\
\text { disciplines, bridging laws of thermodynamics } \\
\text { and social sciences }\end{array}$ \\
\hline
\end{tabular}




\section{Deduction of the driving force of evolution}

The earth's surface has widespread relatively temperate heat streams flowing from the solar, geothermal, and other energy sources. The earth, as a rare habitable planet in astronomy, receives temperate sunlight, which leads to temperate heat streams on the Earth for billions of years [14]. Meanwhile, many sites on the Earth, particularly at hydrothermal vents, have emitted geothermal energy for long periods $[15,16]$. The huge amount of water on the Earth and the atmosphere of the Earth regulate these energy flows through evaporation, diffusion, and rainfall, making them more temperate, last longer, and distributed more widely.

The widespread relatively temperate heat streams on the Earth trigger many physical or chemical changes. According to the second law of thermodynamics (i.e. heat can spontaneously flow from a hotter body to a colder body, see Supplementary Notes) $[17,18]$, stones can spontaneously absorb heat from these heat streams and increase their temperatures, and many CBEs can spontaneously absorb heat from these heat streams to form HHCBEs through chemical bonds, partially because carbon atoms are prone to form covalent and other chemical bonds after absorbing heat [19].

The widespread relatively temperate heat streams on the Earth trigger movement of many materials including wind, water flow, and animal movement. Many CBEs can hence meet and interact with each other to form HHCBEs. For example, lipid molecules can form bilayer membranes and ants can form ant groups in this way, where the energy required for the movement of these CBEs is directly or indirectly from the widespread relatively temperate heat streams on the Earth. The mechanisms for these CBEs to interact to form HHCBEs are given at Section 3.

Although all HHCBEs shall degrade sooner or later, many HHCBEs are relatively stable and can be accumulated. HHCBEs have hence been formed, degraded, regenerated, and accumulated at a myriad of places for billions of years, due to the widespread relatively temperate heat streams on the Earth, which constitutes evolution of CBEs (Figure 1). Therefore, the tendency of CBEs on the Earth to absorb energy to form HHCBEs, is the driving force of evolution.

Initially, the driving force of evolution was from solar energy and geothermal energy. Later, with the increase of organisms on the Earth, biological energy became a source of the driving force of evolution. This is of paramount significance for animals 
which can actively obtain energy and materials from other organisms. Energy from coal, petrol, water flow, and even nuclear power has been utilized by humans for the development of human society.

The above driving force can explain why non-living organic molecules evolved to lives, and why unicellular organisms evolved to multicellular organisms, and why ectotherm animals evolved to warm-blooded animals, because all these three macroevolution events were the processes where CBEs absorbed energy and formed HHCBEs, as driven by thermodynamics. They cannot be well explained with previous theories including natural selection, because none of the processes surely added fitness to CBEs [3-5,10,20,21].

During the whole history of the Earth, the amount and the diversity of HHCBEs including organisms on the Earth are generally increasing [22]. However, meteorite impacts, huge volcano eruptions, long glacial periods, and other catastrophes can destroy the temperate heat streams on the Earth and structures of many organisms [2325]. Consequently, the amount and the diversity of organisms could decline greatly for these catastrophes, sometimes leading to mass extinctions [23-25].

\section{Deduction of the progressive mechanisms of evolution}

\subsection{Three progressive mechanisms}

Three progressive mechanisms of evolution were deduced from the driving force of evolution. The first is termed the driving force mechanism shown with the red arrows in Figure 1, where the driving force of evolution directly raises the amount of HHCBEs and increases the structural complexity and hierarchy of CBEs. The second is termed the structural mechanism shown with the green arrows in Figure 1, where CBEs with increased structural complexity and hierarchy spontaneously obtain some complicated functions, due to interaction inside HHCBEs. For example, when green fluorescence protein is formed by amino acids, it obtains spontaneously the function of emitting green fluorescence, due to interaction of its amino acid components. The third mechanism is termed natural selection shown with the blue arrows in Figure 1, which leads to increase of the diversity and the fitness of CBEs, as detailed below.

\subsection{Natural selection in the CBEET}


As shown in Figure 1, the driving force of evolution leads to formation and accumulation of HHCBEs. The formed HHCBEs shall degrade into LHCBEs sooner or later, due to outer factors (e.g. fire burning) or inner factors (e.g. natural aging) [1,2]. Therefore, lots of CBEs on the Earth are in the cycle of formation and degradation of HHCBEs, leading to regeneration of HHCBEs. Interaction inside an HHCBE constitutes the overall fitness of the HHCBE, which represents the ability of the HHCBE to obtain energy and materials from the environment to regenerate the HHCBE, and the ability of the HHCBE to avoid degradation. Mathematically, some HHCBEs shall survive and some HHCBEs shall disappear, as per their overall fitness constituted by their inner interaction. These natural effects (natural survival of fit HHCBEs and natural elimination of unfit HHCBEs) are defined as natural selection in the CBEET.

Regenerated HHCBEs usually carry some changes. These changes are under natural selection because they influence interaction inside HHCBEs and thus influence the overall fitness of HHCBEs. Those regenerated HHCBEs sharing adequate similarity can be considered as the same species of HHCBEs.

Whether an HHCBE is fit or shall survive or thrive, is determined not only by its inner interaction, but also by its environment. For example, an HHCBE having great fitness in a hot rainforest can be unfit in a cold desert. Moreover, when the environment is comfortable, the fitness threshold of natural selection is low, which can lead to rapid divergence of HHCBEs including species explosion [26], and the fitness threshold of natural selection increases greatly when a catastrophe occurs, which can lead to mass extinction of organisms including those quite fit previously [23-25]. Therefore, although natural selection usually inhibits those changes which reduce fitness (i.e. negative selection), survival of fit HHCBEs includes survival of those HHCBEs carrying changes neutral or harmful in fitness (e.g. thalassemia mutations) if those HHCBEs have adequate fitness in a comfortable environment. In contrast, although natural selection usually supports those changes which add fitness (i.e. positive selection), elimination of unfit HHCBEs includes elimination of HHCBEs carrying changes adding their fitness if their fitness becomes inadequate in a harsh environment.

Natural selection, mutation, genetic drift, and competition have been claimed to be the driving force of evolution [3-5,20,27,28]. These “driving forces" are not directly related to energy, and so they are largely mechanisms or processes of evolution, not the driving force of evolution. The role of energy in biological evolution was highlighted previously $[29,30]$, but energy has not been associated with the driving force of 
evolution. The driving force of evolution in the CBEET deduced from thermodynamics provides energy for evolution of CBEs. Moreover, all the three progressive mechanisms of evolution in the CBEET are derived from the driving force of evolution (Figure 1). Therefore, the driving force of evolution from thermodynamics plays the first leading role in evolution, although natural selection functions extensively in evolution and remains a leading role in evolution.

\subsection{Differences from previous theories}

First, the CBEET definition of natural selection covers non-living CBEs and can be extended to origin of life, while natural selection in previous theories is largely restricted to evolution of organisms.

Second, as shown in Figure 1, natural selection in previous theories is supported by itself and hence suspected of tautology [31], while natural selection in the CBEET is supported by inner interaction of HHCBEs and laws of thermodynamics leading to spontaneous increase and regeneration of HHCBEs, and hence avoids tautology.

Third, natural selection in previous theories usually emphasized fitness in a single aspect, while the CBEET definition of natural selection highlights the overall fitness because the reality is that the survival of an HHCBE is determined by its overall fitness. Therefore, the CBEET allows an organism to have disadvantageous traits, if its overall fitness is adequate. For example, antelopes are less strong than buffaloes to fight against carnivores, but they run fast and have other advantages, making them have adequate fitness in general. This suggests a novel mechanism of sympatric speciation, because multiple combinations of various traits can all constitute adequate overall fitness in occupying the same ecological niche in the same area. Previously, only the mechanism for sympatric speciation targeting different ecological niches in the same area has been proposed, as different ecological niches exert different selection pressures which render organisms evolving towards different directions [3]. Moreover, as detailed in Supplementary Notes, co-action of positive selection and negative selection in various aspects provides a more comprehensive explanation for the evolutionary tempo of punctuated equilibrium, which has been demonstrated in the evolution of many organisms [2-5].

Fourth, as explained above, the CBEET definition of natural selection not only favors changes adding fitness, but also allows disadvantageous traits and changes which are neutral or even harmful in fitness. Hence natural selection in the CBEET 
supports diversity and is less demanding and more inclusive than "survival of the fittest” in Charles Darwin's theory and "gradual replacement with those carrying advantageous mutations" in the Modern Synthesis [1-4]. The CBEET definition reflects the reality correctly, because research advances in molecular biology suggest that most genomic changes are neutral without increase in fitness, and many organisms carry disadvantageous traits and some harmful mutations [3-5,10,12,27].

Fifth, previous definitions of natural selection targeted only inheritable changes, while in the CBEET, genetic mutations, epigenetic changes, and uninheritable changes all influence the overall fitness of HHCEBs, and they are thus all under natural selection. For example, vaccination which is uninheritable makes many animals survive viral infections and pass the relevant natural selection.

Sixth, previous definitions of natural selection assumed that biological mutations occur randomly. Now it has been known that various organisms have the complicated function which makes many mutations occur not randomly, as evidenced in the evolution of microbial genomes and mammalian immunoglobulin genes [9,32].

\subsection{Natural selection targets}

Natural selection in the CBEET targets all hierarchies of CBEs. For example, if a giraffe group is favored by natural selection, the LHCBEs involved in the formation of the giraffe group, including genes, cells, and individuals of the giraffe group are also favored directly or indirectly by natural selection.

Because natural selection "selects" organisms as per their overall fitness which is influenced by all genomic sites and all traits, all genomic sites and all traits are under natural selection [28,33]. Accordingly, natural selection functions extensively in evolution. Moreover, a conserved trait or genomic site without change during a long geological period does not mean that the trait or site is not under natural selection, but likely under strong negative selection [33].

\section{Deduction of the process of evolution}

The driving force of evolution from thermodynamics leads to formation and accumulation of HHCBEs hierarchy by hierarchy. For example, amino acids, nucleotides and other middle organic molecules could not bypass the intermediate hierarchy of large organic molecules to form unicellular organisms, and large organic molecules could not bypass the intermediate hierarchy of unicellular hierarchy to form 
multicellular organisms. Accordingly, as per the backstepping logic (i.e. if hierarchy A exists, the hierarchies lower than hierarchy A should have existed in advance), there should be the following seven major steps constituting evolution of CBEs on the Earth (Figure 1).

Step 7, many animal individuals of the same species interact with each other and form animal groups, which include groups of bees, ants, humans, and many other animals. Animal groups have novel functions which cannot be fulfilled by animal individuals. For example, some ant groups plant fungi for food, which cannot be fulfilled by ant individuals [34]. Some animal groups are eusocial, where some individuals reduce their own lifetime reproductive potential to raise the offspring of others. Many other animal groups are presocial, where the parents take care of their own progenies [35]. Although presocial species are much more common than eusocial species, eusocial species have disproportionately large populations [35].

Step 6, many cells interact with each other and form multicellular organisms, which include fungi, plants, and animals. Multicellular organisms have novel functions which cannot be fulfilled by any cells (e.g. birds can fly faraway which cannot be fulfilled by any cells).

Step 5, many complexes of large organic molecule aggregates interact with each other and form the first batch of unicellular organisms, which are the units having the complicated functions of self-replication via catalysis (for efficiently generating HHCBEs) and self-protection (for efficiently maintaining HHCBEs). The first batch of unicellular organisms emerged at a tiny possibility, and this tiny possibility was realized through the effect of vast spaces and vast time (See Supplementary Notes).

Step 4, many large organic molecule aggregates interact with each other and form complexes of large organic molecule aggregates which, like organelles in the unicellular organisms, have some complicated functions (e.g. synthesis of proteins).

Step 3, many large organic molecules interact with each other and form large organic molecule aggregates (e.g. lipid bilayer membranes and channels allowing ions to pass lipid bilayer membranes) [36]. From this step to the seventh step, energy is not always required to form chemical bonds, but is required for the movement of CBEs to meet and interact with each other.

Step 2, many middle organic molecules (e.g. amino acids, nucleotides, glucose) interact with each other and form proteins, nucleic acids, polysaccharides, and other large organic molecules. Before origin of life, few mechanisms were available to direct 
the synthesis of large organic molecules according to certain orders, and so proteins, nucleic acids, lipids, polysaccharides were produced with few repetitions, and thus the products were of a myriad of diversity. These highly diversified large organic molecules could provide abundant candidates for forming HHCBEs in Steps 3-5. This was beneficial for complexes of large organic molecule aggregates to form unicellular cells at a tiny possibility.

Step 1, many small molecules (e.g. $\mathrm{CO}_{2}, \mathrm{CH}_{4}, \mathrm{H}_{2} \mathrm{O}, \mathrm{H}_{2} \mathrm{~S}$ ) interact with each other and form middle organic molecules (e.g. amino acids, nucleotides, glucose). This step has also occurred on other planets, and lots of middle organic molecules were sent to the Earth by meteorites [37].

Steps 1-5 constitute chemical evolution which is also termed abiogenesis or origin of life. Steps 5-7 constitute biological evolution excluding abiogenesis. Step 7 constitutes animal group evolution including the development of human society.

Steps 1-5 suggest that, before origin of lives, there were five successive and overlapping worlds: the world of small molecules, the world of middle organic molecules, the world of large organic molecules, the world of large organic molecule aggregates, and the world of complexes of large organic molecule aggregates.

Previous evolutionary theories emphasize the special role of RNA (e.g. the world of RNA hypothesis) and some organic molecules with the function of autocatalysis [38,39], while the CBEET highlights collaborative interaction, i.e. collaboration, which constitutes the overall fitness of HHCBEs throughout evolution of CBEs.

Altruism is a special collaboration supporting the production and functions of other entities, and also plays an important role throughout evolution of CBEs. For example, many small molecules spontaneously "sacrifice" themselves to form large organic molecules, and many molecules inside cells "sacrifice” themselves to support spontaneously the replication and functions of nucleic acids, and many cells "sacrifice" themselves in multicellular organisms to support spontaneously the production and functions of germ cells. Many individuals in animal groups "sacrifice” themselves to support spontaneously the birth and functions of other individuals.

Many molecules should obey some rules in cells, and many cells should obey some rules in multicellular organisms. Many individuals in animal groups should obey some rules, including that lion kings should take their responsibility to fight against invaders, and worker bees should work diligently for their groups, and drivers should obey traffic 
rules. Obeying rules constitutes collaboration and altruism inside HHCBEs throughout evolution of CBEs.

As for the seven steps given above, components of HHCBEs are restricted within middle organic molecules (Step 1), large organic molecules (Step 2), large organic molecular aggregates (Step 3), complexes of large organic molecules (Step 4), cells (Step 5), multicellular organisms (Step 6), or animal groups (Step 7). Therefore, freedom of components of HHCBEs should be restricted, and the freedom should increase along with the increase of CBE hierarchies.

Collaboration, altruism, and obeying rules with properly increased freedom inside HHCBEs constitute the overall fitness and biological order of organisms, and they are accumulated through natural selection (Figure 1).

\section{Significance for development of human society}

The CBEET supports the harmonious development of human society. Previous evolutionary theories highlight selfishness, competition, and elimination of those less fit in certain traits [1-5,24]. These notions have been employed to justify authoritarianism, racism, fascism, and Nazism [40]. In contrast, the CBEET not only emphasizes selfish (e.g. self-reproduction and self-protection), fitness, and competition, but also emphasizes diversity, collaboration, altruism, obeying rules with properly increased freedom. Moreover, previous evolutionary theories highlight heritable genetic effects, while the CBEET not only emphasizes heritable genetic effects, but also emphasizes the effects of endeavor to increase fitness, even though the effects are uninheritable. Additionally, previous evolutionary theories highlight advantages of a certain trait, while the CBEET emphasizes not only an important trait, but also all traits. This is important for humans to make comprehensive decisions.

\section{Significance in physics}

The CBEET based on thermodynamics provides novel explanations for origin of life, macroevolution, natural selection, speciation, evolutionary tempos, animal group evolution, and human society development in a comprehensive and comprehensible way, like unlocking the complex building of evolution using the correct key of physics. The CBEET hence bridges physics, biology, and social sciences. Moreover, the CBEET 
refutes thoroughly the wrong notion of negative entropy (negentropy) which has widely skewed sciences for decades [41-47], as detailed in Supplementary Notes.

Acknowledgements: This study has not been supported by any funds so far. The author thanks Jiwang Chen, Meng Yang, Yiqing Chen, and many other people for providing precious comments on this study.

Author contributions: This study is completed by Ji-Ming Chen.

Competing interests: The author declares no conflict of interest.

\section{References}

1. Chen JM. A new evolutionary theory deduced mathematically from entropy amplification. Chin Sci Bul. 2000;45(1):91-96. https://doi.org/10.1007/BF02884912

2. Chen JM, Chen JW. Root of science: the driving force and mechanisms of the extensive evolution. Beijing, China: Science Press, 2000.

3. Futuyma DJ, Kirkpatrick M. Evolution. 4th ed. Sunderland, UK: Sinauer Press, 2017.

4. Huneman P, Walsh DM. Challenging the Modern Synthesis: adaptation, development, and inheritance. Oxford, UK: Oxford University Press, 2017.

5. Preiner M, Asche S, Becker S, et al. The future of origin of life research: bridging decades-old divisions. Life (Basel). 2020;10(3):20. http://doi.org/10.3390/life10030020

6. Pagel M. Natural selection 150 years on. Nature. 2009;457:808-811. https://doi.org/10.1038/nature07889

7. d'Ischia M, Manini P, Moracci M, et al. Astrochemistry and astrobiology: materials science in wonderland? Int J Mol Sci. 2019;20(17):4079. http://doi.org/10.3390/ijms20174079

8. Orgel L. In the beginning. Nature. 2006;439(7079):915-915. https://doi.org/10.1038/439915a

9. Fitzgerald DM, Rosenberg SM. What is mutation? A chapter in the series: How microbes “jeopardize” the modern synthesis. PLoS Genet. 2019;15(4): e1007995. https://doi.org/10.1371/journal.pgen.1007995 
10. Koonin EV. Darwinian evolution in the light of genomics. Nucleic Acids Res. 2009;37(4):1011-1034. http://doi.org/10.1093/nar/gkp089

11. Dickins TE, Rahman Q. The extended evolutionary synthesis and the role of soft inheritance in evolution. Proc Biol Sci. 2012;279(1740): 2913-2921. http://doi.org/10.1098/rspb.2012.0273

12. Casillas S, Barbadilla A. Molecular population genetics. Genetics. 2017;205(3):10031035. https://doi.org/10.1534/genetics.116.196493

13. Walker SI. Origins of life: a problem for physics, a key issues review. Rep Prog Phys. 2017;80(9):092601. https://doi.org/10.1088/1361-6633/aa7804

14. Seager S. Exoplanet habitability. Science. 2013;340(577):577-581. http://doi.org/10.1126/science.1232226

15. Dodd MS, Papineau D, Grenne T, et al. Evidence for early life in earth's oldest hydrothermal vent precipitates. Nature. 2017;543(7643): 60-64. http://doi.org/10.1038/nature21377. PMID 28252057.

16. Proskurowski G, Lilley MD, Seewald JS, et al. Abiogenic hydrocarbon production at lost city hydrothermal field. Science. 2008;319(5863):604-607. http://doi.org/10.1126/science.1151194.

17. DeVoe H. Thermodynamics and chemistry. 2nd Edition. Version 10. http://www2.chem.umd.edu/thermobook/v10-screen.pdf. Accessed on Sep. 20, 2020.

18. Borgnakke C, Sonntag RE. Fundamentals of Thermodynamics. 8th edition. Hoboken, USA: John Wiley \& Sons, 2013.

19. Carbon. https://en.wikipedia.org/wiki/Carbon. Accessed on Sep. 20, 2020.

20. Benton MJ. The Red Queen and the Court Jester: species diversity and the role of biotic and abiotic factors through time. Science. 2009;323(5915):728-732. http://doi.org/10.1126/science.1157719

21. Lynch M. The frailty of adaptive hypotheses for the origins of organismal complexity. Proc Natl Acad Sci U S A. 2007;104(Suppl 1):8597-8604. http://doi.org/10.1073/pnas.0702207104

22. Rohde RA, Muller RA. Cycles in fossil diversity. Nature. 2005;434(7030):208-210. http://doi.org/10.1038/nature03339 
23. Percival LME, Ruhl M, Hesselbo SP, et al. Mercury evidence for pulsed volcanism during the end-Triassic mass extinction. Proc Natl Acad Sci U S A. 2017;114(30):79297934. http://doi.org/10.1073/pnas.1705378114

24. Fields BD, Melott AL, Ellis J, et al. Supernova triggers for end-Devonian extinctions. Proc Natl Acad Sci U S A. 2020;117(35):21008-21010. http://doi.org/10.1073/pnas.2013774117

25. Bader J, Jungclaus J, Krivova N, et al. Global temperature modes shed light on the Holocene temperature conundrum. Nat Commun. 2020;11:4726. https://doi.org/10.1038/s41467-020-18478-6

26. McGee MD, Borstein SR, Meier JI, et al. The ecological and genomic basis of explosive adaptive radiation. Nature. 2020. https://doi.org/10.1038/s41586-020-2652-7

27. Nei M. Mutation-driven evolution. Oxford, UK: Oxford University Press, 2013.

28. Hershberg R, Petrov DA. Selection on codon bias. Annu Rev Genet. 2008;42(1):287299. http://doi.org/10.1146/annurev.genet.42.110807.091442

29. Martin WF, Sousa FL, Lane N. Energy at life's origin. Science. 2014;344:1092-1093. https://doi.org/10.1126/science.1251653

30. Sousa FL, Thiergart T, Landan G, et al. Early bioenergetic evolution. Philos Trans R Soc Lond B Biol Sci. 2013;368(1622):20130088. http://doi.org/10.1098/rstb.2013.0088

31. Hunt T. Reconsidering the logical structure of the theory of natural selection. Commun Integr Biol. 2014;7(6):e972848. https://doi.org/10.4161/19420889.2014.972848

32. Olivieri DN, Mirete-Bachiller S, Gambón-Deza F. Insights into the evolution of IG genes in Amphibians and Reptiles. Dev Comp Immunol. 2020: 103868. https://doi.org/10.1016/j.dci.2020.103868. Epub ahead of print.

33. Chen J, Sun Y. Variation in the analysis of positively selected sites using nonsynonymous/synonymous rate ratios: an example using influenza virus. PLoS One. 2011;6(5):e19996. http://doi.org/10.1371/journal.pone.0019996.

34. Styrsky JD, Eubanks MD. Ecological consequences of interactions between ants and honeydew-producing insects. Proc Biol Sci. 2007;274(1607):151-164. http://doi.org/10.1098/rspb.2006.3701 
35. Nowak M, Tarnita C, Wilson E. The evolution of eusociality. Nature. 2010;466:10571062. https://doi.org/10.1038/nature09205

36. Levy E, Erba E, Robinson C, et al. Assembly reflects evolution of protein complexes. Nature. 2008;453:1262-1265. https://doi.org/10.1038/nature06942

37. Follmann H, Brownson C. Darwin's warm little pond revisited: from molecules to the origin of life. Naturwissenschaften. 2009;96(11):1265-1292. http://doi.org/10.1007/s00114-009-0602-1.

38. Kauffman SA. Approaches to the origin of life on the Earth. Life (Basel). 2011;1(1):3448. http://doi.org/10.3390/life1010034.

39. Robertson MP, Joyce GF. The origins of the RNA world. Cold Spring Harb Perspect Biol. 2012;4(5): a003608. http://doi.org/10.1101/cshperspect.a003608.

40. Rudman LA, Saud LH. Justifying Social Inequalities: The role of social Darwinism. Pers Soc Psychol B. 2020;46(7):1139-1155. https://doi.org/10.1177/0146167219896924.

41. Schrödinger E. What is life - the physical aspect of the living cell. Cambridge, UK: Cambridge University Press, 1944.

42. Viswanadham CR. Entropy, evolution and living systems. Nature. 1968;219(5154):653. https://doi.org/10.1038/219653b0.

43. Woolhouse HW. Entropy and evolution. Nature. 1967;216(5111):200. https://doi.org/10.1038/216200a0.

44. Jennings RC, Belgio E, Zucchelli G. Photosystem I, when excited in the chlorophyll Qy absorption band, feeds on negative entropy. Biophys Chem. 2018;233:36-46. https://doi.org/10.1016/j.bpc.2017.12.002.

45. Bejan A. Evolution in thermodynamics. Appl Phys Rev. 2017;4(1):011305. https://doi.org/10.1063/1.4978611.

46. Boltzmann L. The second law of thermodynamics. In Theoretical physics and philosophical problems (pp. 13-32). Dordrecht, Netherlands: Springer, 1974.

47. Wilson JA. Increasing entropy of biological systems. Nature. 1968;219(5153):534-5. https://doi.org/10.1038/219534a0. 


\section{Supplementary Notes}

\section{Note 1. Fundamentals of thermodynamics $[17,18]$}

The first law of thermodynamics: increase of internal energy of a closed system is equal to the work the surroundings gives to the system plus the heat the surroundings gives to the system.

The second law of thermodynamics: heat can spontaneously flow from a hotter body to a colder body, and cannot spontaneously flow from a colder body to a hotter body; the entropy of an isolated system never decreases over time.

The third law of thermodynamics: the entropy of a system approaches a constant value as its temperature approaches absolute zero, and the entropies of perfect crystals at absolute zero temperature are zero.

The Boltzmann formula of entropy: $S=k \cdot \operatorname{Ln} \Omega$, where $S, k$, and $\Omega$ denote entropy, a constant, and microstates (i.e. possible microscopic configurations), respectively. This formula suggests that the entropy of a closed system is only determined by its microstates which are related to the physical and chemical states of the system, particularly the temperature of the system, as higher temperature means more rapid thermal motion of molecules, more microstates, and greater entropy.

The Clausius inequality of entropy: $d S \geq \delta Q / T$, where $d S, \delta Q$, and $T$ denote changes of the entropy of a closed system, heat absorbed by the system from the surroundings, and absolute temperature, respectively. This inequality suggests that: first, the entropy of a closed system shall increase if the system absorbs heat from the surroundings, and the entropy of a closed system shall decrease if the system dissipates heat to the surroundings; second, the increase of the entropy of a closed system shall be greater if the system is colder when it absorbs the same amount of heat from the surroundings.

\section{Note 2. Evolution, entropy, and negentropy}

Evolution of CBEs leads to increase of biological order. This seems contrary to the second law of thermodynamics which means the entropy of an isolated system never decreases over time and entropy represents chaos in thermodynamics [17,18,41]. Some 
scientists assumed that the entropies of organisms are low because organisms have much order, and organisms keep low entropy through absorbing low-entropy matter and discharging high-entropy matter. Hence the notion of negative entropy (negentropy) was established [41,42], which prevails in common people and scientists [41-44].

We believe that the notion of negentropy is wrong in this aspect, because it is incorrect to assume that all kinds of order, including biological order, are equal to the low-entropy thermodynamic order, for the following seven reasons. First, taking a dog as an example and a close system, if this dog is dying in the snow, its biological order declines as it is dying, and its thermodynamic order increases because its entropy declines with heat being lost from the dog to the surroundings, as per the Clausius inequality of entropy $[17,18]$. Second, a live dog is a warm and moving system with many microstates, and it has hence high entropy, compared with perfect crystals at absolute zero temperature which have the lowest entropy ( $S=0$ ) and the highest thermodynamic order, as per the Boltzmann formula of entropy and the third law of thermodynamics $[17,18]$. Third, as deduced in Section 3, biological order is established through long-term natural selection, rather than a short-time metabolic effect of negentropy. Fourth, during the period that a fertilized egg grows into a mature dog, the biological order of this organism changes little, while its entropy increases billions of times, as per the additive property of entropy $[17,18]$. Fifth, contrary to the notion of negentropy, the entropy of what we absorb can be greater than the entropy of what we discharge, which is clearly demonstrated by this special example: the materials absorbed by a fetus for rapid growth in the uterus are much more than the materials discharged by the fetus, and thus the entropy of the materials absorbed by the fetus is greater than the entropy of the materials discharged by the fetus. Sixth, under certain inherent mechanisms, some high-entropy systems, such as organisms, choruses, armies, airplanes, and skyscrapers, can demonstrate some kinds of order, which is distinct from the cold, low-entropy and crystal-like thermodynamic order (Box 1). Seventh, lives rely on entropy rather than negentropy, because lives rely on movement rather than immobilization of their inner components [45]. Ludwig Boltzmann, who created the Boltzmann formula of entropy, also pointed that animate beings struggle for entropy which becomes available through the transition of energy from the hot sun to the cold earth [46]. This view was inherited by the CBEET. Although negentropy has been criticized previously [41,47], the above seven reasons refute negentropy thoroughly and clearly. 
Box 1. Another widespread notion pertaining to entropy is also incorrect.

A pile of books placed neatly is frequently claimed to have less entropy than the same pile of books placed messily. In effect, the entropy of this pile of books changes little no matter whether they are arranged neatly or messily, as the books do not absorb heat from the surroundings or dissipate heat to the surroundings through the arrangement. The macroscopic chaos of the books we observe with eyes is different from the chaos of the books at the level of microscopic particles (i.e. microstates, which determine the entropy of the pile of books).

\section{Note 3. Mechanism for punctuated equilibrium}

Previously geographical isolation was employed to explain the evolutionary tempo of punctuated equilibrium which has been demonstrated in the evolution of many organisms [2-5]. Here co-action of positive selection and negative selection provides a more comprehensive explanation for punctuated equilibrium, as detailed in Figure S1, which suggests that, beyond geographical isolation, climate or ecological changes can also trigger significant changes in traits in short geological periods.

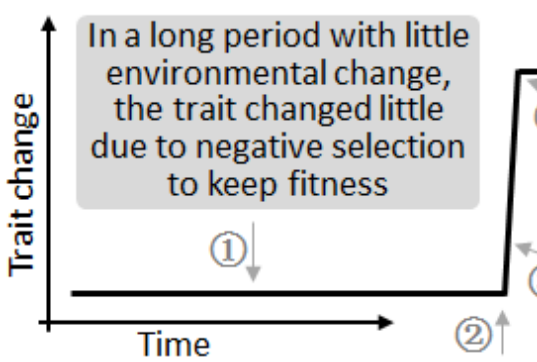
(5)

In a long period with little environmental change, the trait changed little due to negative selection to keep fitness

(4) Further change in the trait shall add no fitness to the organism, due to co-action of positive selection in some aspects and negative selection in some other aspects

The trait was changing under positive selection to meet the environmental requirements, and the change always accumulated significantly in a short geological period

The environment changed significantly due to spatial changes (e.g. entering a new place) or climate/ecological changes (e.g. a forest becoming a savanna)

Figure S1. Reasons for the evolutionary tempo of punctuated equilibrium

\section{Note 4. How could life emerge at a tiny possibility through}

\section{abiogenesis?}

The Earth 3.8 billion years ago could be much warmer than the current Earth, and most of the then Earth could be covered with warm water containing abundant of small, middle, large organic molecules, large organic molecule aggregates, and complexes of 
large organic molecule aggregates. If these CBEs on the whole Earth tried to form unicellular organisms for one billion times every hour, and the successful possibility was only 10-15, then over 8 thousand unicellular organisms could emerge through abiogenesis within one million years. 